\title{
Research on Embedded GUI Technology and Its Application in Control System of Engineering Machinery
}

\author{
Yan $\mathrm{Hou}^{1}$, Jinxia Huang ${ }^{2, *}$ \\ ${ }^{1}$ College of Clinical Medical Laboratory, Jiamusi University, Heilongjiang Province, P. R. China, \\ 154007 \\ ${ }^{2}$ College of Information electronic Technology, Jiamusi University, Heilongjiang Province, \\ P. R.China 154007 \\ *email:329223252@qq.com
}

Keywords: Embedded GUI technology; engineering machinery control system; application research

\begin{abstract}
This paper makes deep analysis on design process and main technology of embedded GUI; it makes detailed design and realization on its key part-Graphic Device Interf. Ace, meanwhile it combines with embedded GUI development software to develop one set of embedded GUI system with strong function and beautiful interface for one actual engineering machinery control system.
\end{abstract}

\section{Introduction}

This paper makes deep analysis on design process and main technology of embedded GUI; it makes detailed design and realization on its key part-Graphic Device Interf. Ace, meanwhile it combines with embedded GUI development software to develop one set of embedded GUI system with strong function and beautiful interface for one actual engineering machinery control system.

Firstly, this paper makes demand analysis on embedded GUI, illustrates the main technology of mainstream GUI, as for multi-task communication part, it gives detailed algorithm flow and program code. Secondly, it makes deep research on graphic device inerf. ace in GUI system, it makes detailed design and realizes it on basic drawing module, text module as well as output and input layers. Thirdly, it constructs embedded Linux operation system in PCI04 industrial control panel, including system boot, kernel porting and construction of file system. And it allocates and installs MiniGUI on this development platform; it makes graphic user interface development and porting of the input engine by combining with actual engineering machinery control system. In the end of this paper, it makes performance test on embedded GUI system by self-writing software and expert test tool, and it demonstrates its effectiveness by comparing it with other embedded GUI system.

\section{Status analysis on embedded UGI technology}

\section{Construction and layer of embedded UGI.}

The usual GUI system design adopts system structure of vertical stratification; the general structure is indicated by figure 1. By adopting vertical stratification system structure, GUI system has clear layer, the interface between layer and layer is clear, easy and abstract. 


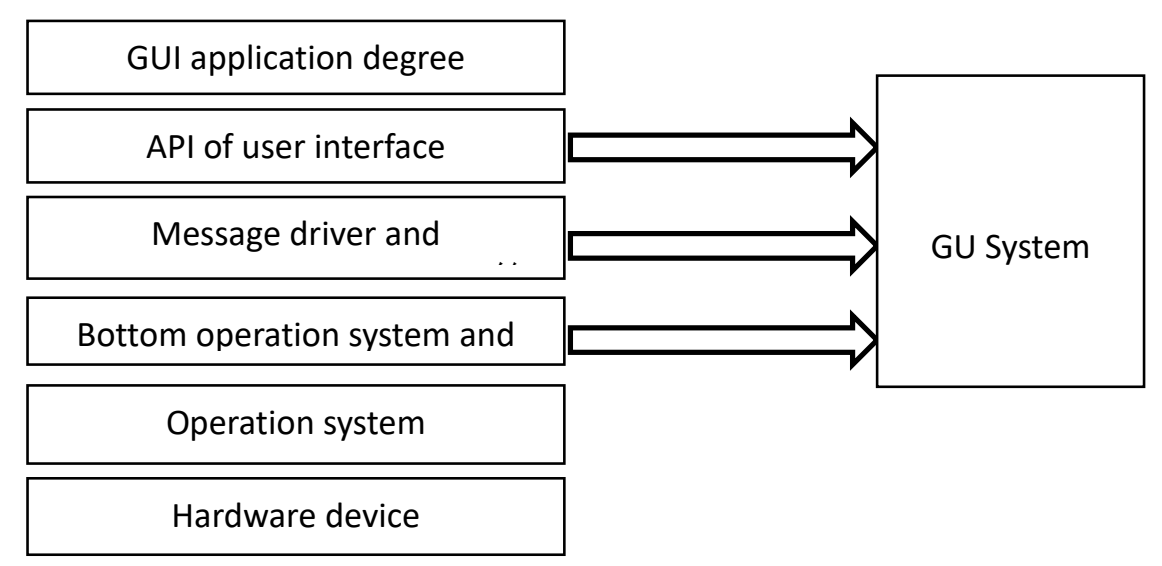

Figure 1 The usual system structure of GUI

At present, the mainstream technology most adopts microkernel idea, stratification system structure and idea of technology faces with object combining with designing embedded GUI system. Microkernel technology makes core module design of GUI highly abstract, reliable, its high abstract can greatly enhance portability and extendibility. It makes high abstract on similarity of GUI and forms one simplified GUI system kernel-GUI microkernel. This kernel only provides basic operation principle of GUI, high abstract interface of operating GUI environment and high abstract interface hardware support demanded by GUI. Microkernel of GUI basically makes no work relates to special software and hardware environment, neither it makes any direct support on user interface, neither it makes any direct support on user interface, it just makes support on detailed realization and expansion of GUI system. This kind of high abstract is very important for design of embedded system, the universal application of microkernel technology is to make system kernel small and reliable, strengthen portability and expansion of system. Etc. As for support of file system, network etc, it all regards it as application disposal of microkernel, microkernel will make dispatch on them, they are completely configurable. The system structure of adopting microkernel idea to design system structure of GUI figure 2:

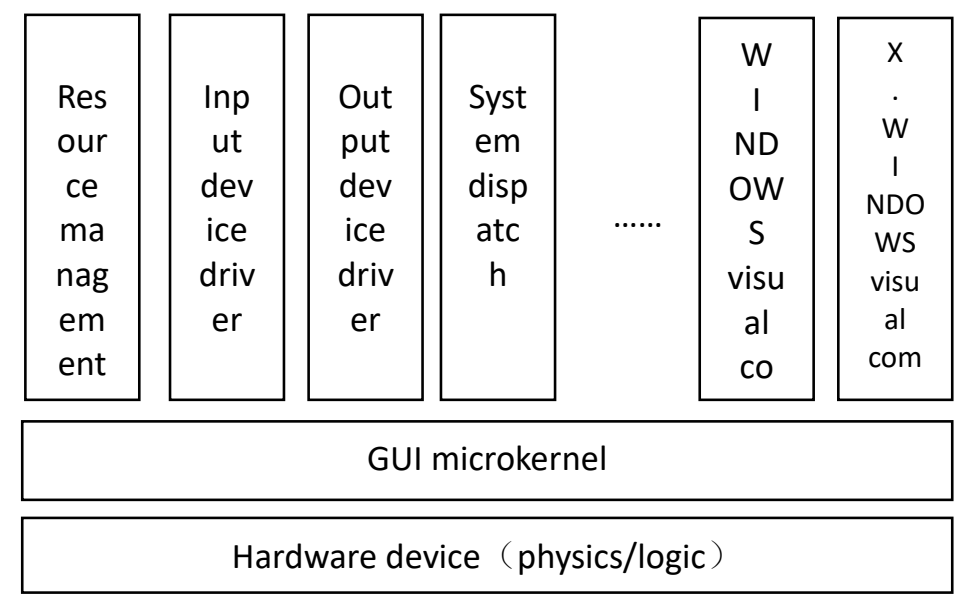
is indicated by

Figure 2 GUI system structure of adopting microkernel

The system of adopting microkernel, the modules above (outside) microkernel can make configuration according to requirements. For example, some GUI systems are driven by communication event, it only needs to output device display and it can not make operation by input device, this kind of system is not used to support input device, generally speaking, while requirements on real-time of output device is very high. Users can realize components and selfdefined components with different visual perception standard, it can be conveniently added to system and it can also easily delete the unnecessary modules from system. So GUI system designed by idea of microkernel has good portability and tailorability, it can also make configuration according to different applications. Microkernel itself is unrelated to detailed application and special operation system; it just needs to make correction and transplantation on modules such as input 
device driver module, output device driver and system dispatch module etc, so system has good portability. In the field of industrial control, it urgently needs GUI of light grade, these systems has high requirements on real-time, while it also has high requirement on GUI interface and its performance. While GU of Windows or Linux on the present universal PC operation system needs to occupy plenty of system resources, it can not meet industrial control requirement. So at present, there are many systems adopt relatively simple methods on DOS to realize graphic user interface. So these systems have urgent demand on GUI of light grade. Embedded system generally makes different design according to application occasion and control cost, the external design supported by them is different, so it has different demand on GUI. Some systems only need some graphic function. At present, this needs our GUI system must have characteristic of being tailored. Now, both China and abroad have developed the universal graphic user interface system used in embedded environment, the matured mainly has XWindows , MiniGUI, Microwindows, openGUI, QT/Embedded etc. The overall structure of XWindow system is indicated by the following figure 3: $\mathrm{x}$ Client

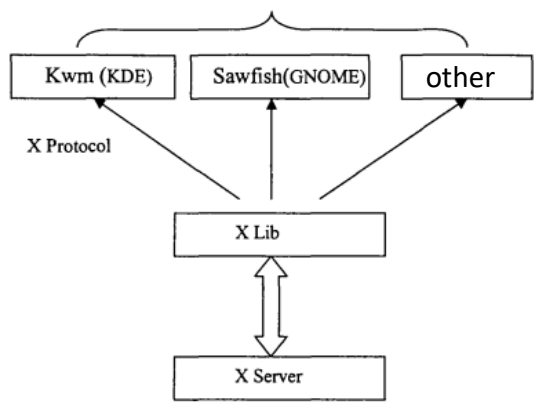

Figure 3 XWindow structure graphic in Linux/UNXI

The structure adopted by XWindow system has good expansion and transplantation etc. But at the same time, it also makes XWindow can only become to be one application in UNXI, while it can make high integration with kernel of operation system, this makes system based on XWindow has defects such as large scale, burden and low efficiency etc. In order to obtain portability of application program, many factories have tried to make shortening of XWindow system to make it can be operate on the embedded system.

\section{Analysis on driver mechanism of embedded UGI.}

The event driver mechanism of GUI system is mainly message driven mechanism. From message mechanism, this GUI system is divided into 3 sub-systems: graphic processing sub-system, input device sub-system and output device sub-system, every sub-system uses graphic processing sub-system as the core; they are relatively independent and related, which is indicated by the following figure 4 :

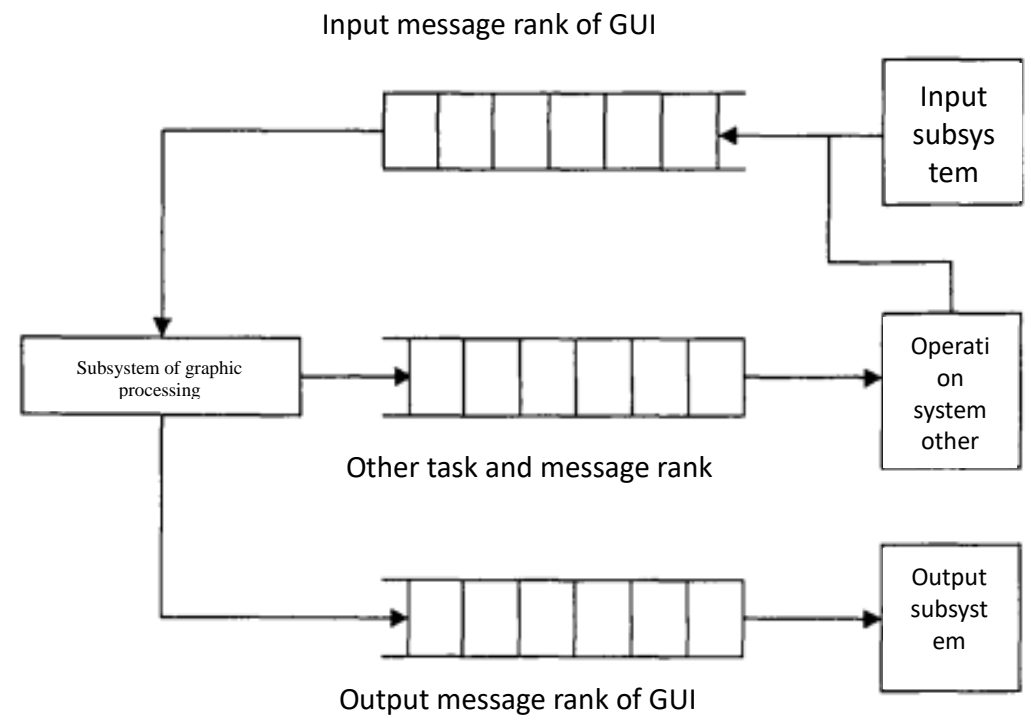

Figure 4 Message processing mechanism of general GUI system 
Message processing mechanism is used mainly to solve the event processing problem based on hardware interruption in program design of early period. Because occurrence of interruptive event can not be predicted and is abrupt, when there are many application waiting and process interruptive event, it will have problems. Message mechanism can well solve multi-application and design problems in the event driven, form the simple and reliable method to process relations among many systems, components within system and components. At present, GUI systems on the most operation system adopt message mechanism.

\section{Embedded GUI technology and its application in engineering machinery control system}

\section{Realization of embedded Linux on the panel.}

Start of embedded Linux system is related to 3 software parts in the system: boot loader, kernel and init.

Usually, boot loader is the first program operated when hardware system is started; its main task is to make hardware initialization of low grade, and then gives control right of CPU to start code. The implemented kernel start code in the beginning is different with the difference in system structure of target system, its task is to make related initialization of system structure, set proper operation environment of $\mathrm{C}$ Language, and then dispatches the entry function of kernel. For example, on the PC machine, LILO or GIIIIB, which is the boot loaded. Afterwards, advanced function of kernel initialization, such as memory management module, process management module and hardware device etc, and then it will load root file system and finally start init process. The remaining system start tasks are completely completed by init process in user space.

\section{Embedded GUI design.}

(1)Construction of MiniGUI running environment. The software environment of this host is as follows: Redhat. According o demand of real project, the running environment requirement of MiniGUI is as follows: firstly is to ensure video card of host is VESA compatible, secondly is to ensure Linux kernel includes FrameBuffer support, VESAFrameBuffer driver program, thirdly is whether FrameBuffer is activated or not.

(2) Install MiniGUI

(3) Graphic user interface development based on MiniGUI. Graphic user interface is in the middle layer of software structure in the whole system. As for the embedded Linux operation system in the bottom, it is one application program operated above it, while as for the function module at the top layer, it is one software running platform, all the function modules are dispatched and terminated by this platform, and display the running result to users by platform.

(4) Input engine transplantation. In the embedded system based on Linux, input device may only has little touch screen and keys, as for graphic engine, processing of input device has no uniform interface, under this condition, it is very important to provide one abstract input layer.

\section{Coveralls design of engineering machinery control system.}

This engineering machinery control system mainly includes 3 parts, the overall structure of system is indicated by figure 4, the man tasks of this paper is focus on the front control sub-system of boot loader, including pattern selection of system hardware platform, construction of embedded Linux operation system and realization of embedded GUI design. 


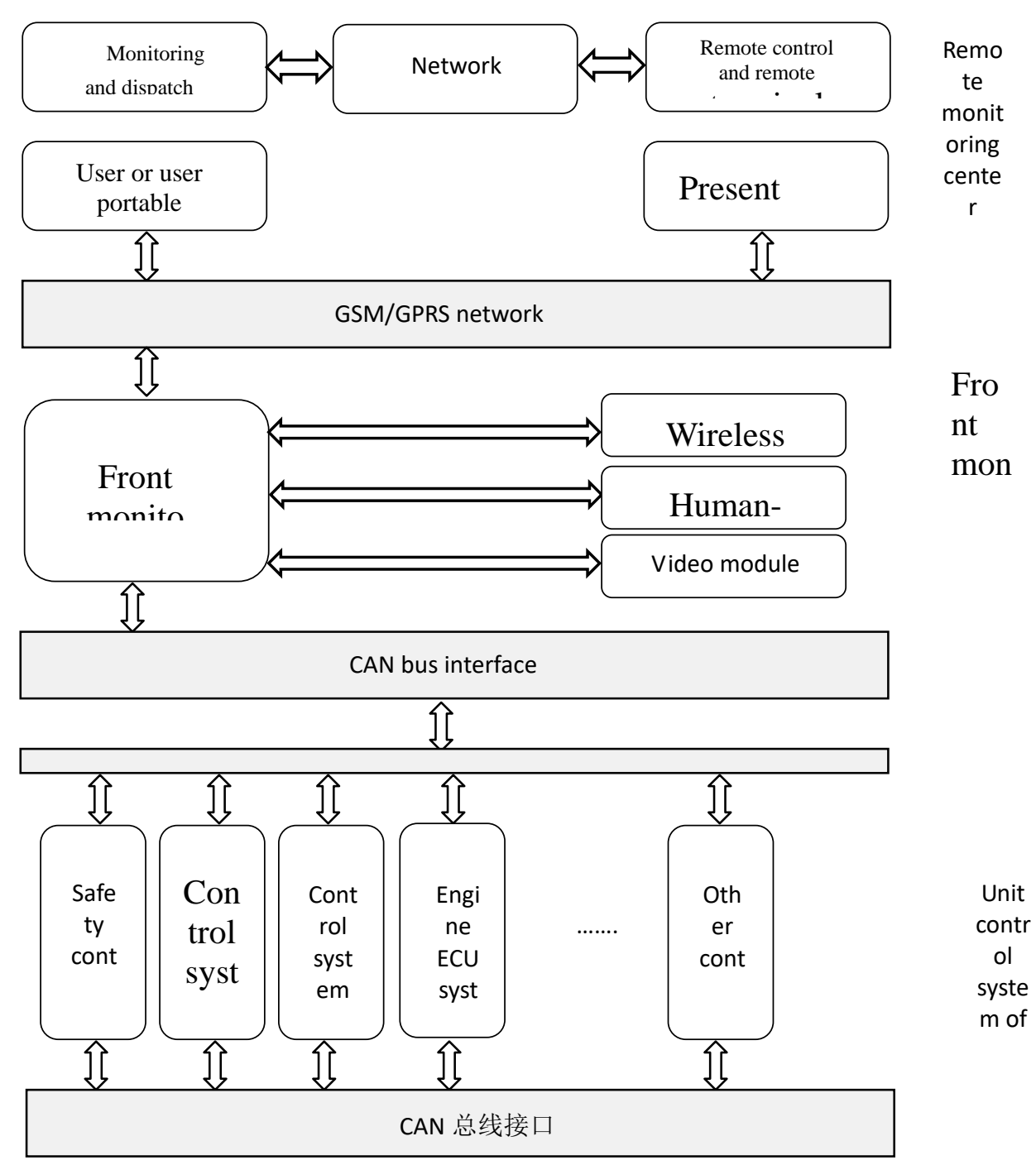

Figure 5 Overall structure of control system

The monitoring system of the front end of boot loaded is regarded as one processing terminal of information communication, it uses CANBUS and GPRS(General Packet Radio Service) to realize upload and transmission of data, and it provides good user interface, as for data and information of every terminal control module in engineering machinery, it provides one visualized management, $\mathrm{p}$ provides video processing function, realizes working condition of engineering machinery and video display of blind area for human eyes, it transmits video information to remote monitoring center by GPRS. Through the above analysis, the front monitoring system of boot loader must have the following functions: (1) CAN communication function, it can meet requirement of real-time data communication with control unit at the bottom. (2) GPRS communication function, it can meet requirement of real-time data communication with remote monitoring center. (3) Video processing function, it meets requirement of timely displaying working condition graphic. (4) Friendly graphic user interface, it is convenient for application of operators. In the aspect of non-function demand, it has the following points: (1) because it is used in the control of portable engineering machinery and its working environment is very severe, so it must ensure the reliable operation of system. (2) It must ensure production cost of product is not so high. (3) It must ensure the development progress of product conforms to requirement.

\section{Establishment on movement and control equation for engineering machinery device}

According to the detailed structure relation of engineering machinery device movement bar and definition of coordinate system, it can get the following formula: 
The formula of position vector component is as follows:

$$
\left\{\begin{array}{l}
T_{X}=\cos (q 1)^{*} u 1+\sin (q 1) *(d 4-d 5) \\
T_{Y}=\sin (q 1) * u 1-\cos (q 1)^{*}(d 4-d 5) \\
T_{Z}=U 2+11
\end{array}\right.
$$

Inverse solution of engineering machinery device kinesiology:

$$
\left\{\begin{array}{l}
x_{0}=\cos (q 1) *\left(l_{1} \cos (q 2)-l_{2} \cos (q 2+q 3)+l_{3} \cos (q 2+q 3+q 4)-l_{4} \cos (q 2+q 3+q 4+q 5)\right. \\
\left.+l_{5} \cos (q 2+q 3+q 4+q 5+q 6)\right) \\
y_{0}=l_{1} * \sin (q 2)-l_{2} * \sin (q 2+q 3)+l_{3} * \sin (q 2+q 3+q 4)-l_{4} * \sin (q 2+q 3+q 4+q 5)+l_{5} * \\
\sin (q 2+q 3+q 4+q 5+q 6) \\
z_{0}=\sin (q 1) *\left(-l_{1} * \cos (q 2)+l_{2} * \cos (q 2+q 3)-l_{3} * \cos (q 2+q 3+q 4)+l_{4} * \cos (q 2+q 3+q 4+q 5)\right. \\
\left.-l_{5} * \cos (q 2+q 3+q 4+q 5+q 6)\right)
\end{array}\right.
$$

\section{Coordinate mapping formula:}

$\mathrm{xViewport}=((\mathrm{xWindow}-\mathrm{xWinOrg}) * \mathrm{xViewExt} / \mathrm{xWinExt})+\mathrm{xViewOrg}$

y Viewport $=((y W i n d o w-y W i n O r g) * y$ ViewExt / yWinExt $)+y$ ViewOrg

Under MM TEXTm mapping environment, mapping formula can be simplified as follows:

$\mathrm{x}$ Viewport $=x$ Window-xWinOrg $+x$ ViewOrg

y Viewport $=(y W i n d o w-y W i n O r g+y$ ViewOrg

\section{Conclusions}

With the universal application of expert computer system such as embedded system, virtual reality, visualization of scientific computation and quick development of multi-media technology, new human-machine alteration technology is emerging and becomes increasingly mature, more humanized alteration way is emphasized by the public. Embedded GUI technology has simple, beautiful and visualized operation, advantages based on service custom-made resources; it gets universal application in more and more application fields. Of which, in the aspect of engineering machinery, engineering machinery control system of the new generation not only needs to realize integrated operation and intelligent control, but also needs possibly provide friendly, direct operation interface without information blind side for site operators and background management personnel. Demand analysis before developing embedded GUI, it includes description of general structure, standard function and performance description, afterwards it analyzes several mainstream technology of embedded GUI system in key points, meanwhile it develops research on technology layer for multi-task communication of GUI, and it gives development flow and algorithm idea. As for the key layer of GUI technology, it makes analysis, realization and design on graphic user interface, of which including design and connection of basic drawing module, text module, image module as well as input and output layers. As for pattern selection of hardware platform and construction of operation system, it fully displays merits of Linux taking up little space and resources etc, it realizes one micro operation system on the PCI04 working control panel. It installs and configures MilliGUI in the constructed embedded operation system, makes design and program development of graphic user interface on the host, transplants target file, base file after development to working control panel by cf card, it completes the final commissioning integration and transplantation of engine.

\section{Acknowledgment}

This work is supported by The Education Department of Heilongjiang Province Natural Science Project Research on rapid detection and control system of urine microalbumin test paper,Science and technology research project of Jiamusi University Project $13 Z 1201570$. 


\section{Reference}

[1] Xing Weiwei, Fan Shangchun. Development Platform of Embedded Graphic User Interface [J].Journal of Beijing University of Aeronautics and Astronautics, 2003, 09:829-833.

[2] Zhan Jinyu, Xiong Guangze, Sun Ming. Realization Plan of One Kind of Embedded GUI Software Structure [J]. Journal of University of Electronic Science and Technology of China, UESTC, 2003, 01:89-93.

[3] Liu Jingmeng, Wang Tianmiao, Liu Miao, Wei Hongxing, Tao Wei, Wang Xiaojun. Embedded Technology and Its Application in Engineering Machinery Monitor [J].Engineering Machinery, 2003, 06:5-8+1.

[4] Xie Chao, Li Shanping. Embedded GUI Based on LINUX [J].Computer Engineering and Application, 2003, 09:135-137.

[5] Hong Xiaobin,Liu Guixiong, Xun Qingui,Chen Tiequn. Touching GUI Realization on IP Model Intelligent Measurement and Control Device of the New Generation [J].Automaton of Manufacturing Industry, 2009, 02:71-74.

[6] Automatics and Computer Technology [J].Electronics Abstract of Chinese Wireless, 2009, 01:173-233. 